\title{
Establishing the torsional rigidity of a rotation module responsible for the flexion-extension motions of the elbow
}

\author{
Tudor Deaconescu* and Andrea Deaconescu \\ Transilvania University of Braşov, Department of Industrial Engineering and Management, Bd. \\ Eroilor 29, Braşov, Romania
}

\begin{abstract}
The paper presents and discusses a system devised for the mobilisation of the elbow including a pair of agonist-antagonist pneumatic muscles aimed mainly at ensuring the accuracy of the rotation angle. This system is of SISO type (single input - single output) with the variable $\Delta p$ (the pressure by that one of the muscles is charged symmetrically in the detriment of the other) as its input value. Further discussed are the dependency of the equilibrium angle on $\Delta p$ and the possibility of controlling the torsional rigidity by adjusting the sum of the feed pressures.
\end{abstract}

\section{Introduction}

At present, due to the diversification, miniaturisation and improvement of pneumatic components, the area of applicability of compressed air is expanding continuously. In addition to the obvious applications like flammable or explosive work environments, compressed air is used in the medical field, like in the motion-based rehabilitation of reduced or lost functional capacities of patients, consequently to congenital or acquired affections. The main advantages of pneumatic drives are in this latter case: reduced risk of accidents, a non-polluting work environment, the possibility of adjustment of forces and velocities over a wide range, and most importantly, a specific feature known as compliance. Compliance, the inverse of rigidity is an actuator's characteristic of allowing deviations from a certain position of equilibrium when the system is subject to the action of exterior forces.

Many robotic applications, like prosthetics, medical rehabilitation, assembling require variable stiffness actuators (VSAs) or, under a different designation adjustable compliant actuators (ACAs). These types of actuators allow minimising large forces that occur in cases of collision (shock) thus ensuring safe interaction with the user, and have the capacity of storing energy in elastic elements of passive type and of releasing it, respectively [1].

An adjustable compliant actuator is capable of ensuring the adaptability of the actuated system to a concrete working situation that may differ from the initially envisaged one. A mechanical system is defined as adaptive when it has the capacity of adequately responding to new situations within the limitations given by the mechanical band width determined by the system's elasticity and inertia, as well as by the internal friction. In such a system the

\footnotetext{
* Corresponding author: tdeacon@unitbv.ro
} 
presence of sensors or complex controllers is not necessary, as it is the mechanical system itself that provides the required adaptive behaviour [2-3].

Actuators are adjustable compliant when their stiffness is not constant, which is the case when the dependency between the developed force and the displacement is non-linear. Pneumatic muscles - bio-inspired actuation systems - fit this description, due to their adaptive compliant behaviour, materialized by the possibility of continuous variation of the stiffness [3]. The utilization of pneumatic muscles in the construction of medical rehabilitation systems still is in an early stage, because of the relatively recent emergence of these actuators.

In this context this paper studies the torsional rigidity and compliance of a rotation module responsible for the flexion-extension motions of the elbow. The rotation module is actuated by a torsion motor consisting of a pair of agonist-antagonist pneumatic muscles.

\section{Construction of the torsion motor designed for the elbow rehabilitation equipment}

The purpose of rehabilitation is combatting pain, improving the amplitude of joint mobility, blood flow, and muscle tonus.

In the case of the elbow, the main objective of post-traumatic recovery is to restore the normal motion amplitude, achievable by the healthy segments prior to trauma or surgical intervention. For flexion-extension of the elbow, the normal motion amplitude is of $140^{\circ}$ ($90^{\circ} \ldots+50^{\circ}$ ) (Figure 1).

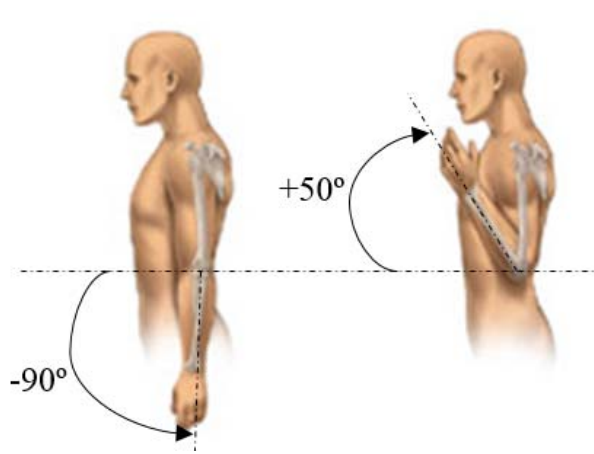

Fig. 1. Limits of elbow flexion-extension.

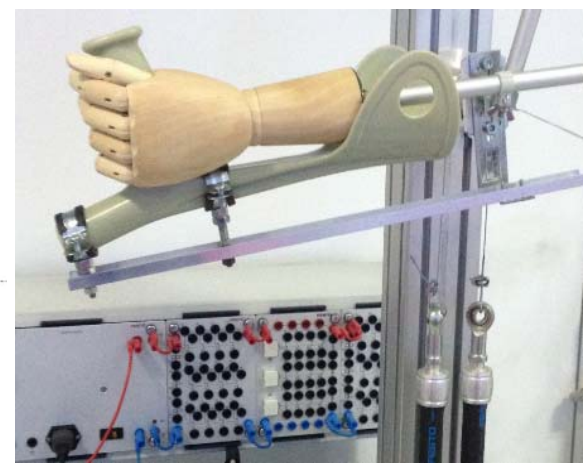

Fig. 2. Construction of the elbow rehabilitation equipment.

Figure 2 shows the construction of the equipment developed for the recovery of elbow flexion-extension motion amplitude. The flexion-extension motion is achieved by a torsion motor consisting of a pair of agonist-antagonist pneumatic muscles. Their role is to generate, based on their antagonist operation, a rotation of the desired amplitude $\left(140^{\circ}\right)$, as well as to ensure the equilibrium of a given position of the actuated system. Another requirement this system has to meet is compliance, namely to allow small deviations from the position of equilibrium when unforeseen exterior forces occur.

The torsion motor is obtained by linking two pneumatic muscles that operate in agonist-antagonist mode. While one of the muscles rotates a load, the other one acts like a brake, also ensuring that the motion is stopped in a certain position of equilibrium. The upper ends of the two pneumatic muscles are tied each by two flexible steel cables to a pulley that materializes the elbow joint (Figure 3). By inflating one muscle and at the same time deflating the other a rotation in one or the other direction is generated. 
The discussed equipment deploys two DMSP-20-300N-RM-CR pneumatic muscles (manufactured by Festo, Germany), with a $20 \mathrm{~mm}$ interior diameter and a $300 \mathrm{~mm}$ active length [4].
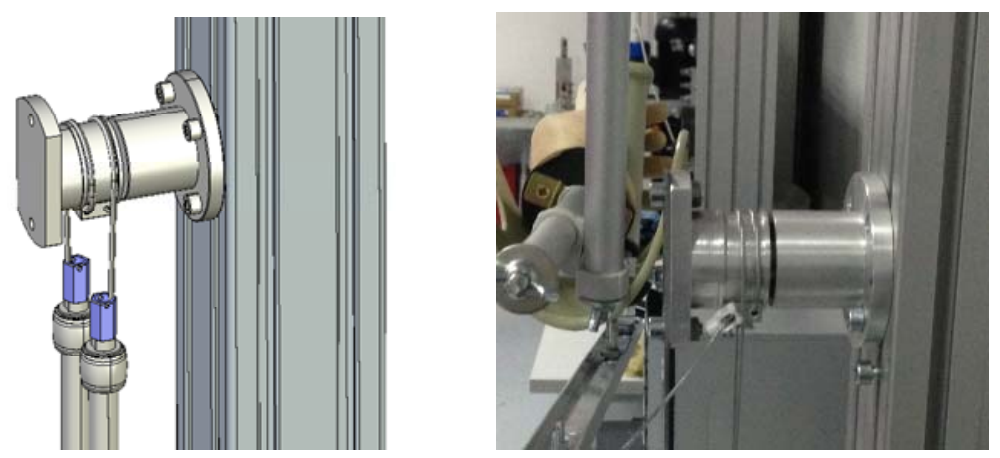

Fig. 3. Construction of the rotation module.

\section{Computation of the torsional rigidity and the compliance of the rotation module}

For the two pneumatic muscles being assembled for agonist-antagonist operation, the torque developed in the rotation module is given by equation (1) [5]:

$$
T=K_{1} \cdot\left(p_{1}-p_{2}\right)-K_{2} \cdot\left(p_{1}+p_{2}\right) \cdot \theta
$$

where $p_{1}$ and $p_{2}$ denote the feeding pressures of the two muscles, and $K_{1}$ and $K_{2}$ are coefficients, the values of which depend on the dimensional characteristics of the utilised pneumatic muscles (in this case $\mathrm{K}_{1}=24.52$ and $\mathrm{K}_{2}=15.83$ ) [5].

When torque $T$ is zero a position of equilibrium of the joint can be defined:

$$
\theta_{e c h}=\frac{K_{1} \cdot\left(p_{1}-p_{2}\right)}{K_{2} \cdot\left(p_{1}+p_{2}\right)}
$$

Starting from equation (1) the torsional rigidity of the joint and its compliance, respectively, can be computed by equations (3) and (4):

$$
\begin{aligned}
& k=\frac{d T}{d \theta}=\left|-K_{2} \cdot\left(p_{1}+p_{2}\right)\right| \\
& C=k^{-1}=\left|-\frac{1}{K_{2} \cdot\left(p_{1}+p_{2}\right)}\right|
\end{aligned}
$$

The agonist-antagonist actuation mode of the rotation module is of MIMO (multiple input-multiple output) type, with the two feed pressures $p_{1}$ and $p_{2}$ as input quantities and the output quantities being the static torque $T$, the rotation angle $\theta$ and the torsional rigidity $k$.

In the case of the system driving the joint to carry out flexion and extension, the main aim is to obtain an accurate as possible rotation angle $\theta$. Having thus set the control of the angular position as the priority, the actuation system has to be transformed from a MIMO into a SISO (single input-single output) one, with angle $\theta$ as its single output quantity. The variable $\Delta p$ will be considered as input quantity, namely the pressure by that one of the muscles is charged and the other one discharged symmetrically.

Starting from the moment when both pneumatic muscles have been simultaneously charged to pressure $p_{0}=p_{\max } / 2=3 \mathrm{bar}$, rotation in one or the other direction entails feeding 
compressed air to one of the muscles and releasing the same quantity $\Delta p(\Delta p=0 \ldots 3$ bar) from the other. Thus:

$$
\begin{aligned}
& p_{1}=p_{0}+\Delta p \\
& p_{2}=p_{0}-\Delta p
\end{aligned}
$$

where from equation (1) becomes:

$$
T=2 \cdot K_{1} \cdot \Delta p-2 \cdot K_{2} \cdot p_{0} \cdot \theta
$$

If torque $T$ is zero, a position of equilibrium of the joint can be defined:

$$
\theta_{\text {ech }}=\frac{K_{1} \cdot \Delta p}{K_{2} \cdot p_{0}}=0.516 \cdot \Delta p
$$
$\Delta p:$

Figure 4 illustrates the dependency of the equilibrium angle on the pressure difference

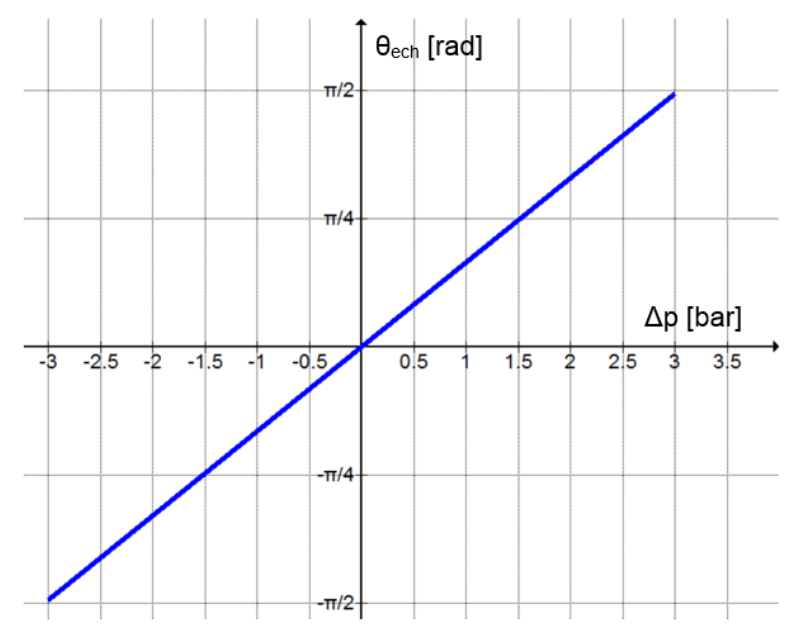

Fig. 4. Variation of angle $\theta_{\text {ech }}$ versus $\Delta p$.

The torsional rigidity of the joint and its compliance can be computed in this case by equation (9) and (10):

$$
\begin{gathered}
k=\frac{d T}{d \theta}=-2 \cdot K_{2} \cdot p_{0} \cdot 10^{-1}=-9,498 \mathrm{~N} \cdot \mathrm{m} / \mathrm{rad} \\
C=-\frac{1}{2 \cdot K_{2} \cdot p_{0} \cdot 10^{-1}}=-0.105 \mathrm{rad} / \mathrm{N} \cdot \mathrm{m}
\end{gathered}
$$

In equations (8) and (9) it can be observed that a certain position of equilibrium can be obtained by varying the pressure difference $\Delta p$, while the torsional rigidity of the joint remains constant.

As regarding the torsional rigidity and the compliance of the rotation module, equations (3) and (4) show that these can be adjusted by modifying the sum $\left(p_{1}+p_{2}\right)$ according to the graphs in Figures 5 and 6.

With the increase of the feed pressures the torsional rigidity will have greater absolute values, yielding the conclusion that for a better positioning precision the two muscles should be fed higher pressures. Such a situation, however, causes compliance to diminish and a less "soft" interface with the patient. As in rehabilitation exercising positioning precision is less 
important, and it is the compliance of the system that has to be maximised, it follows that the pneumatic muscles have to be fed at small pressures.

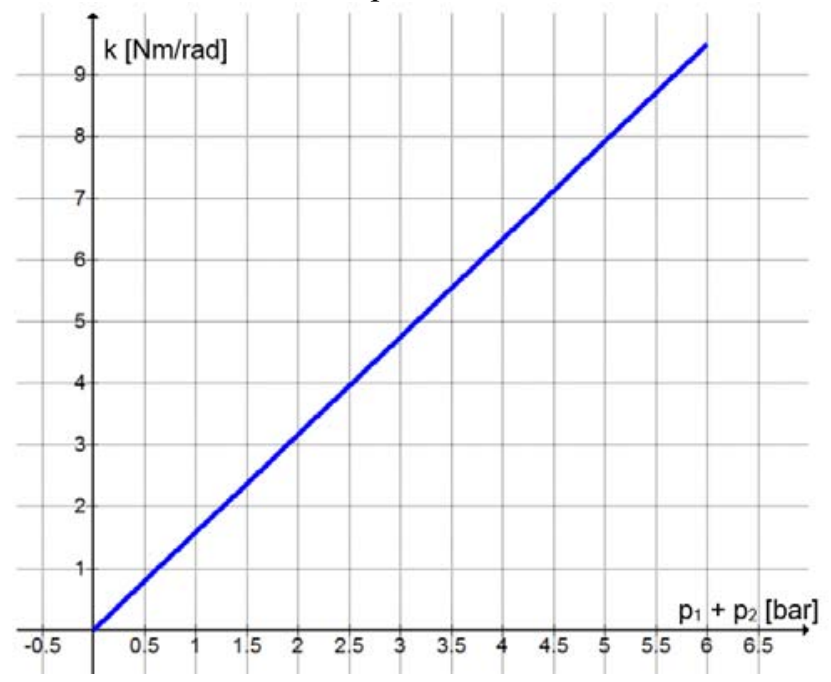

Fig. 5. Adjusting torsional rigidity by modifying the sum of the feed pressures.

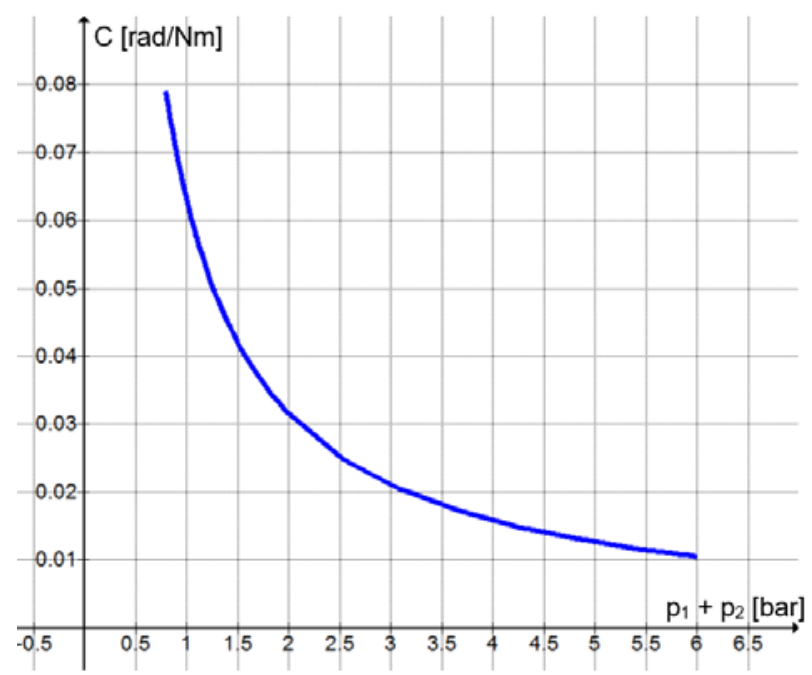

Fig. 6. Adjusting compliance by modifying the sum of the feed pressures.

Figure 7 shows the variation of the torques in two distinctive cases: in one case the sum of the pressures is equal to 6 bar entailing a high torsional rigidity of the system, while in the other case $p_{1}+p_{2}=2$ bar leading to a diminished rigidity and enhanced compliance.

It has to be noted that if the system caused to leave its position of equilibrium and rotates by a quantity $\Delta \theta$, it tends to return to its initial position due to the onset of a return torque computed by equation (11):

$$
T_{\text {rev }}=-K_{2} \cdot\left(p_{1}+p_{2}\right) \cdot \Delta \theta=-k \cdot \Delta \theta
$$

The above equation and Figure 7 show that as the torsional rigidity $k$ increases, the value of the return torque will be greater. 


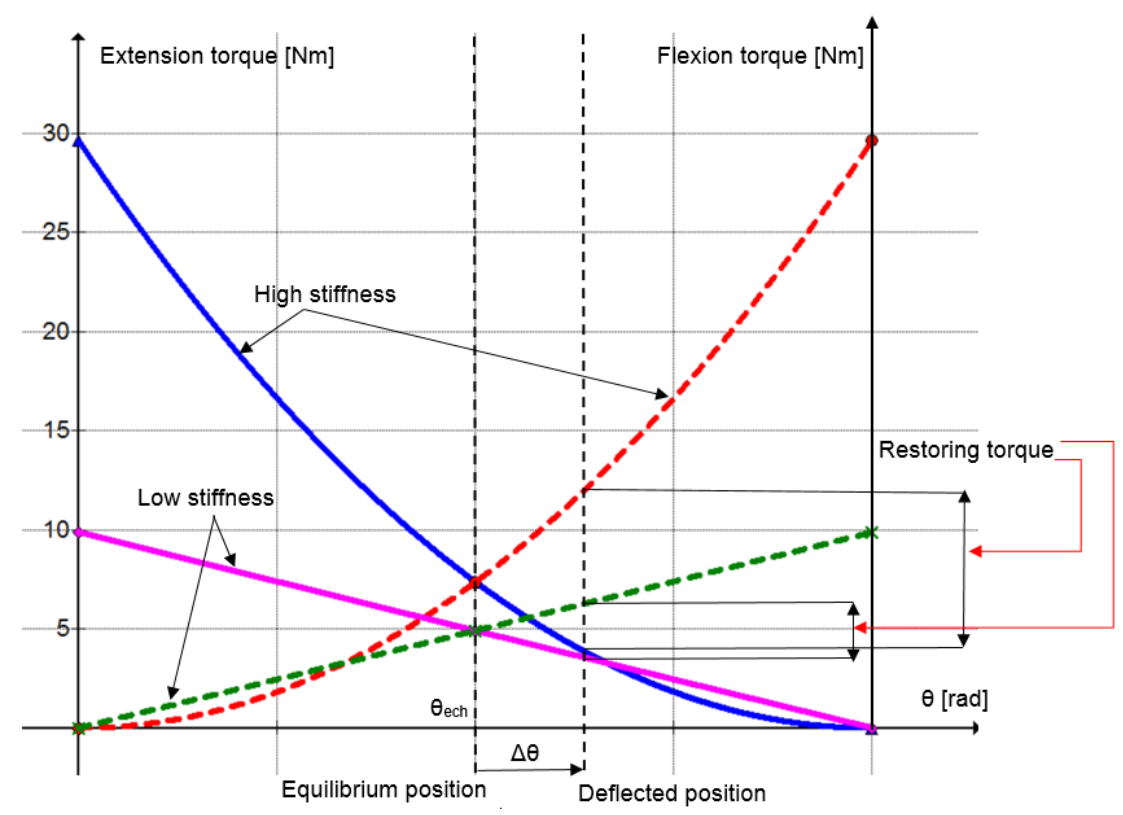

Fig. 7. Co-activation of the pneumatic muscles such as to obtain two levels of torsional rigidity.

The above Figure shows that feeding low pressure to the two pneumatic muscles yields a smaller rigidity of the torsion motor. This entails a better compliant behaviour of the actuated system, rendering it more user-friendly. This property is essential for medical rehabilitation equipment.

\section{Conclusion}

The paper presents a methodology for the computation of the torsional rigidity and the compliance of a rotation module designed for conducting the flexion-extension motions of the forearm by means of a medical rehabilitation equipment. According to the pursued scope, namely obtaining a high precision or rather a high compliance, the feed pressures of the two pneumatic muscles can be determined. The conclusion of the performed analysis is that in order to obtain a high compliance of the rehabilitation equipment the low pressures are required for feeding the actuation system.

\section{References}

1. R. Van Ham, T.G. Sugar, B. Vanderborght, K.W. Hollander, D. Lefeber. IEEE Robotics \& Automation Magazine 16(3), 81-94 (2009)

2. D. Petkovic, M. Isac, N.D. Pavlovic, L. Zentner. Applied Mechanics and Materials 162, 316-325 (2012)

3. T. Deaconescu, A. Deaconescu. Strojniški vestnik - Journal of Mechanical Engineering 63(4), 225-234 (2017)

4. Festo, Fluidic Muscle DMSP/MAS, https://www.festo.com/rep/en_corp/assets/pdf/info_501_en.pdf Accessed: 12.12.2016

5. G. Vetrice, Report 2, PhD Thesis Transilvania University of Brasov (2018) 